\title{
Choosing the right bar: a complex problem
}

\section{Mai M Morimoto \& Aman B Saleem}

Mai M. Morimoto and Aman B. Saleem are at the UCL Institute of Behavioural Neuroscience, Department of Experimental Psychology, University College London, London, UK.

e-mail: aman.saleem@ucl.ac.uk

Inputs toof the central complex, the navigation centre in Drosophila, are strongly modulated by the visual stimulus history. These history effects carry forward to bias turning behavioural choice when flies choose between two visual stimuli.

[AU: Adjust figure panel citations to reflect the new panel order. Note that panels should be cited in order; if not possible, cite the figure as a whole first.] Navigation can have many strategies, entailing varying degrees of complexity in planning one's route. One of the simpler strategies, with minimal planning, is beacon navigation, which involves moving towards a salient landmark. This simple strategy is in common use across species from humans to flies. Consistent with such simple a navigation strategyies, flies naturally[AU: ok?] turn towards a dark bar in their visual field, a behaviour referred to as stripe-fixation ${ }^{1}$. What if there are two bars? Flies are generally unbiased ${ }^{2}$ in their choice as to which bar to turn to. In this issue of Nature Neuroscience, Shiozaki and Kazama report the fascinating discovery that this choice between two bars can be biased by stimulus history ${ }^{3}$. They find that flies choose to turn to a bar in a new location (Figure 1). In a parallel study, Sun et al. use a technical innovation, in vivo two-colour two-photon imaging, to record activity of inputs to the central complex, the navigation centre of insects. They observe effects of stimulus history that could explain the biases in behavioural responses ${ }^{4}$.

Even with a simple nervous system, flies display some intriguing visually guided navigational behaviours. For example, when a fly is placed in a cylindrical arena with two dark bars opposite to each other, it walks back and forth between the bars ${ }^{5}$. Even when a bar disappears for a short time, the fly continues to walk in the direction of the absent bar. This suggests that flies remember the position of the bar for a short time, navigating using visual short-term memory ${ }^{6}$. Other studies using associative learning tasksparadigms studies[AU: ok? or tasks, models, procedures?] also support the case for such visual[SA1] memory based navigation. A fly version of the Morris water maze shows that flies can quickly learn to use visual patterns on an arena wall to locate a cool tile in an otherwise aversively hot tiled arena7. In both of these studies, ring neurons of the ellipsoid body ofin the central complex (whose neurites form a ring in the ellipsoid body) were found to be necessary for the visually guided navigation behaviour ${ }^{6,7}$. 
The insect central complex also represents navigation-related signals. Specifically, some neurons of the ellipsoid body represent heading direction in flies ${ }^{8,9}$. This representation is similar to that of head-direction cells in rodents ${ }^{10}$ or bats ${ }^{11}$. Given that the central complex represents navigational information and is involved in visually guided short-term memory, it is a promising locus in which to study how visual information can be transformed into memory- guided navigation. To investigate this, onewe would first need a system to record neural activity during visual-memory- guided navigation.

Shiozaki and Kazama ${ }^{3}$ used a virtual reality system that was controlled by the wing-beat patterns of a tethered fly. A wing-beat pattern that corresponds to a leftward turn makes the visual stimulus move rightward (while the fly remains fixed), ): a virtual left turn. As expected from stripefixation behaviour ${ }^{1}$, flies turned towards a bar when one bar was presented (Figure $1 \mathrm{~b}$ ). When there was a choice between two bars, one on the left and one on the right, flies were equally likely to turn to either of the bars (Figure 1a,c). The authors then investigated the effects of stimulus history by cueing the fly with a bar before giving them the choice of two bars. Each trial had three periods: cue, delay and choice (Figure 1d). During the cue period a bar was presented on one of the sides. After a delay period when the screen was left blank, two bars were presented in the choice period.

Curiously, they found that cueing on one side biased flies turn to the opposite side during the choice period. For example, if the bar was presented on the left (cued side) during the cue period, flies would turn to the right (uncued side) in the choice period. This bias in choice bias lasted for delay periods as long as 8 seconds, suggesting that this behaviour could be supported by short-term memory.

Given previous reports of the involvement of ring neurons [AU: Can you introduce and briefly explain this term above, and also cite the relevant reference(s) here? (Renumber refs. as needed.)] of the central complexellipsoid body in spatial short-term memory ${ }^{6,7}$, Shiozaki and Kazama ${ }^{3}$ investigated whether the same neurons were necessary for the stimulus historydependent choice bias. When they silenced a subset of ring neurons, flies lost the bias to turn towards the uncued side but continued to show the stripe-fixation behaviour. This suggests ring neurons are involved in the history- dependent bias in the two-bar choice behaviour.

Sun et al. ${ }^{4}$ provide a possible mechanism for such choice behaviour by measuring the stimulus- history- dependent activity of these ring neurons. First, using functional connectivity mapping, the authors found that the main pathway from the fly's visual centre to the central complex was via a region called the bulb (Figure 1e). The bulb is organized into small microglomeruli, each containing one presynaptic and one postsynaptic partner (TuBu and ring neurons, respectively; 
Figure 1e). This monosynaptic organization in the microglomerulus makes it an ideal place to look at information transfer between a pair of neurons. In a technical tour de force, Sun et al. ${ }^{4}$ succeeded in spectrally separating the emission spectra of two calcium indicators, jRGECO (red) and GCaMP (green), enabling two-colour two-photon imaging. Sun et al. ${ }^{4}$ recorded responses of neurons in the bulb to visual stimuli while flies passively viewed them. Neurons recorded on one side of the brain generally responded strongly to a bright bar presented on the same side. This response was reduced when a second bar was presented at the same time on the opposite side, an effect termed contralateral suppression. Sun et al. ${ }^{4}$ next investigated the effect of stimulus history on the responses and found a clear effect: response to the two-bar stimulus decreased even further when it was preceded by a single bar on the ipsilateral side. The effect of stimulus history was much more prominent in the ring neurons in comparison to their pre-synaptic partners in the bulb, the TuBu neurons[AU: in comparison to what?].

Based on the observed effects of stimulus history and contralateral suppression, the authors propose a model that has an excitatory component from ipsilateral stimuli and an inhibitory component from contralateral stimuli. Their model captures the history effects they observed in the neurons. If one assumes that the ring neurons mediate the turning behaviour of flies, the predictions of the model are in line with the history-dependent behavioural biases observed by Shiozaki and Kazama ${ }^{3}$. This suggests that the mechanism observed by Sun et al. ${ }^{4}$ might underlie the history effects in the two-bar choice behaviour.

Shiozaki and Kazama ${ }^{3}$ also monitored neural activity across the microglomeruli of the bulb during the history-dependent two-bar choice task using single-colour two-photon imaging. They imaged both the dorsal and ventral regions, and report interesting differences between them. (Sun et al. ${ }^{4}$ focused their analysis on the dorsal region of the bulb.) The dorsal region responded preferentially to a bar on the ipsilateral side during the cue period (consistent with the results of Sun et $\left.a l .{ }^{4}\right)$. Occasionally, there was increased activity during the following delay period-perhaps a trace of the previous stimulus. The ventral region instead was more responsive to a bar on the contralateral side during cue period. The activity of the ventral region also tracked the turning behaviour of the fly throughout the behavioural task. The authors suggest that the dorsal and ventral regions are functionally distinct parallel pathways that feed information about visual cues and self-motion to the central complex. Supporting this view, anatomical evidence showed that ring neurons in dorsal and ventral bulb project to distinct regions of the ellipsoid body (outer versus inner regionsring[AU: "ring" is being used in $\mathbf{2}$ different senses here, which may be confusing; can you revise?]; Figure 1e). This functional divide between the dorsal and ventral bulb is consistent with 
recent findings by Omoto et al. ${ }^{4}$, who establish TuBu neurons innervating the dorsal and ventral bulb as two separate populations originating in developmentally distinct lineages ${ }^{12}$.

Sun et al. ${ }^{4}$ demonstrate the power of two-color two-photon imaging in vivo, greatly expanding the ability to simultaneously record functional activity of genetically distinct neurons. This work sets an example for how such a method can be readily implemented in genetically tractable model systems. Both Sun et $a .^{4}$ and Shiozaki and Kazama ${ }^{3}$ have discovered surprising effects of stimulus history on the responses of neurons in the input region of the central complex, as well as on the two-bar choice behaviour. This type of history effect could be a functional analogue of working memory in vertebrates - an ability that is required for solving cognitive tasks, including navigation.

While the two studies are complementary, there are some striking discrepancies between their results on behaviour and neural activity. First, while the neurons of the bulb prefer bright contrast bars, the stripe-fixation behaviour is preferentially elicited by dark contrast bars. Second, the time course of the history effect (or memory) seems to differ greatly between them. The behavioural effects observed by Shiozaki and Kazama ${ }^{3}$ are substantially more than three times longer than the history effects in neural activity reported by Sun et $a l .{ }^{4}$ [AU: It might be helpful to quantify the comparison here (give actual time values); $8 \mathrm{~s}$ was given above for the former, but no value has been given for the latter]Finally, the two studies were conducted under different behavioural states, quiescent versus flying. These differences provide many open questions for future investigations in the central complex. After all, choosing the right bar has always been a complex problem.

Figure 1 History of visual stimulus modulates neural activity of the bulb and biases a fly's behavioural choice.

(a) Shiozaki \& Kazama ${ }^{3}$ used a two-bar choice task in which flies were allowed to turn[AU: ok? to avoid implying a trained behavior] towards either the right or left bar.

(b) Flies turned robustly towards a single bar presented in their visual field.

(c) When flies were presented with two bars, they were equally likely to turn towards either bar.

(d) If a single bar was presented in the cue period followed by a delay period (up to $8 \mathrm{~s}$ ), the fly's subsequent choice of the bar was biased to the novel bar position.

(e) Upper panel : Sun et al. ${ }^{4}$ identify a neural pathway from the visual center (optic lobe) to the navigation center (central complex) of the fly. Lower panel : Shiozaki \& 
Kazama ${ }^{3}$ and Sun et al. ${ }^{4}$ both recorded neural activity of the bulb (dotted outline), an input region to the central complex. Sun et $a l .{ }^{4}$ simultaneously recorded connected neuron pairs (green shades, TuBu neurons; magenta shades, ring neurons) using in vivo two-photon two-color imaging and found history effects of visual stimuli. Shiozaki \& Kazama ${ }^{3}$ observed differences in task-related activity between the dorsal and ventral bulb, from which ring neurons project to different regions of the ellipsoid body of the central complex.

\section{References}

1. Reichardt, W. \& Wenking, H. Optical detection and fixation of objects by fixed flying flies. Naturwissenschaften 56, 424-425 (1969).

2. Sareen, P., Wolf, R. \& Heisenberg, M. Attracting the attention of a fly. Proceedings of the National Academy of Sciences of the United States of America 108, 7230-7235 (2011).

3. Shiozaki, H.M., Kazama, H. TBA. Nature neuroscience TBA, TBA (2017).

4. Sun, Y., et al. Neural signatures of dynamic stimulus selection in Drosophila. Nature neuroscience 20, 1104-1113 (2017).

5. Gotz, K.G. Visual guidance in Drosophila. Basic Life Sci 16, 391-407 (1980).

6. Neuser, K., Triphan, T., Mronz, M., Poeck, B. \& Strauss, R. Analysis of a spatial orientation memory in Drosophila. Nature 453, 1244-1247 (2008).

7. Ofstad, T.A., Zuker, C.S. \& Reiser, M.B. Visual place learning in Drosophila melanogaster. Nature 474, 204-207 (2011).

8. Seelig, J.D. \& Jayaraman, V. Neural dynamics for landmark orientation and angular path integration. Nature 521, 186-191 (2015).

9. Turner-Evans, D., et al. Angular velocity integration in a fly heading circuit. Elife 6 (2017).

10. Taube, J.S. The head direction signal: origins and sensory-motor integration. Annual review of neuroscience 30, 181-207 (2007).

11. Finkelstein, A., et al. Three-dimensional head-direction coding in the bat brain. Nature 517, 159-164 (2015).

12. Omoto, J.J., et al. Visual Input to the Drosophila Central Complex by Developmentally and Functionally Distinct Neuronal Populations. Current biology : CB 27, 1098-1110 (2017). 